\title{
Effect of Breathing on Exposed Lung Volumes and Doses in Patients with Breast Carcinoma Receiving Radiotherapy*
}

\author{
Evren Ozan Göksel ${ }^{1 \#}$, Evrim Tezcanli², Melahat Garipağaoğlu ${ }^{2}$, Öznur Şenkesen ${ }^{1}$, \\ Halil Küçücük ${ }^{1}$, Meriç Şengoz ${ }^{2}$, Nuran Beşe ${ }^{3}$, Işık Aslay ${ }^{4}$ \\ ${ }^{1}$ Department of Radiation Oncology, Acibadem Kozyatagi Hospital, Istanbul, Turkey \\ ${ }^{2}$ Department of Radiation Oncology, Acibadem University, Istanbul, Turkey \\ ${ }^{3}$ Oncology Institute, Istanbul University, Istanbul, Turkey \\ ${ }^{4}$ Department of Radiation Oncology, Cerrahpasa Medical School, Istanbul University, Istanbul, Turkey \\ Email: \#evrenozangoksel@yahoo.com, tezcanlievrim@gmail.com,melahat.garipagaoglu@gmail.com, \\ osenkesen@gmail.com, halilkucucuk@gmail.com,msengoz@asg.com.tr, \\ nuranbese@superonline.com, isik.aslay@gmail.com
}

Received March 8, 2013; revised April 10, 2013; accepted May 27, 2013

Copyright (C) 2013 Evren Ozan Göksel et al. This is an open access article distributed under the Creative Commons Attribution License, which permits unrestricted use, distribution, and reproduction in any medium, provided the original work is properly cited.

\begin{abstract}
Introduction: This study evaluates the changes in the lung volume (LV) exposed radiation during the breath cycle and whether these volume differences have an effect on both lung and target doses in breast carcinoma patients. Material and Methods: Ten patients with left breast carcinoma underwent breast conservative surgery or mastectomy receiving radiotherapy (RT) (breast or chest wall and regional lymph nodes) were included. For this study, planning computerized tomography (CT) images were obtained during deep inspiration (DI) and end of expiration (EE), besides free breathing (FB) to simulate breath cycles. Three-dimensional conformal or intensity-modulated RT planning was done to obtain dose-volume information using CT series taken FB, DI and EE. The treatment plan was done with FB images and exported to the DI and EE scans and re-calculated. Volume changes and calculated dose differences according to breath cycles were compared. Results: There were significant differences in the whole LV, ipsilateral LV and contralateral LV between FB-DI and EE-DI while no significant difference was seen between FB and EE. V20 was lower during DI than FB and EE but the difference was not significant. There was no significant variation in whole breast dose although significant dose variations were observed in mean MI, supraclaviculary and level III axillary lymph node doses between breath cycles. Conclusion: Breath cycle had no significant effect on whole breast dose although significantly changed regional lymph node doses in patients with breast carcinoma receiving whole breast and regional lymph nodes radiotherapy. V20 dose was lower during DI than FB and EE, but the difference was not significant.
\end{abstract}

Keywords: Radiotherapy; Treatment Planning; Breast Carcinoma; Lung Volume; Lung Dose; Breath Cycle

\section{Introduction}

Radiotherapy (RT) is an indispensable treatment option for most patients with breast carcinoma because of its benefits of local disease control and on improving survival rates. However, $5 \%-30 \%$ of the ipsilateral lung is exposed to radiation during RT [1,2]. Radiation induced lung side effects can occur during and after completion of radiotherapy [3]. These side effects can manifest with different clinical symptoms such as radiation pneumonitis (shortness of breath) or physical changes such as

${ }^{*}$ This study presented as a poster in UROK 2010 and ESTRO 29.

"Corresponding author. bronchial stricture; they can also be detected using imaging techniques e.g. density changes in computerized tomography (CT) and perfusion-ventilation defects in SPECT scintigraphy $[3,4]$. According to the normal tissue control probability model, the incidence of clinical symptoms and severity of disease depend on both radiation dose and the volume of exposed lung [5-8]. In order to reduce the radiation exposed hearth and lung volume, sophisticated radiotherapy techniques such as respiratory-gated radiotherapy have been used $[9,10]$.

The targets in the treatment of breast carcinoma comprise the chest wall, breast and/or regional lymph nodes. 
Tangential fields are often used to irradiate the breast and/or chest wall in order to protect the lungs and heart. Tangential beam axis passes through target-lung-target. A decrease in the lung tissue density results in an increase in electron scattering, which subsequently leads to an increase in the radiation dose in areas beyond and in front of the lung $[11,12]$. Therefore, tissue density heterogeneity correction is considered during RT planning in clinical practice. RT planning is based on a series of CT images obtained without breath control (FB), which might not include all anatomical changes during a whole breath cycle. In other words, this CT series may not include peak phases of breathing, namely the end of expiration (EE) and deep inspiration (DI). To our knowledge, there is only one study examined whether organ-tissue motion during breathing has an effect on the target dose in patients with breast carcinoma. They reported target dose was not significantly affected by breath cycles. However they looked for whole breast dose. Their study did not include regional lymph node doses [13].

This study evaluates the changes in the lung volume exposed radiation during the breath cycle and whether these volume differences have an effect on both lung and target doses in breast carcinoma patients.

\section{Patients and Methods}

Ten consecutive patients with left breast carcinoma who underwent breast conservative surgery (BCS) or mastectomy $(\mathrm{M})$, receiving radiotherapy (breast, chest wall and regional lymph nodes) were included.

All patients were positioned supine on a carbon fiber breast board having a fixed base with adjustable tilting, and a body cast was fabricated to immobilize the patient's shoulder, in order to ensure daily set-up accuracy. Planning CT images were obtained from the upper neck to the upper abdomen at $3 \mathrm{~mm}$ intervals with a multidetector 16 slices CT (Siemens Sensation 16 Erlangen, Germany), while the patient was in the treatment position on a flat tabletop. Because lung volumes vary with breathing and planning images taken during one particular moment of breath cycle such as expirium, inspirium, and these images do not represent whole breath cycles. Therefore the estimation of lung side effects based on DVH parameters is not precise. In this study, to simulate the lung volumes changes during the respiratory cycle, DI and EE series were also acquired, apart from FB image series, in same sequence to obtain unchanged DICOM coordinates as used in previous studies [14,15]. DI and EE image series were registered to FB, according to DICOM coordinates using ECLIPSE version 8.6 (Varian, Palo Alto, USA) radiotherapy planning system (Figure 1). The target and organs at risk (OAR) volumes were contoured by primary Radiation Oncologist; on 3 different sets of images taken, according to RTOG breast con-

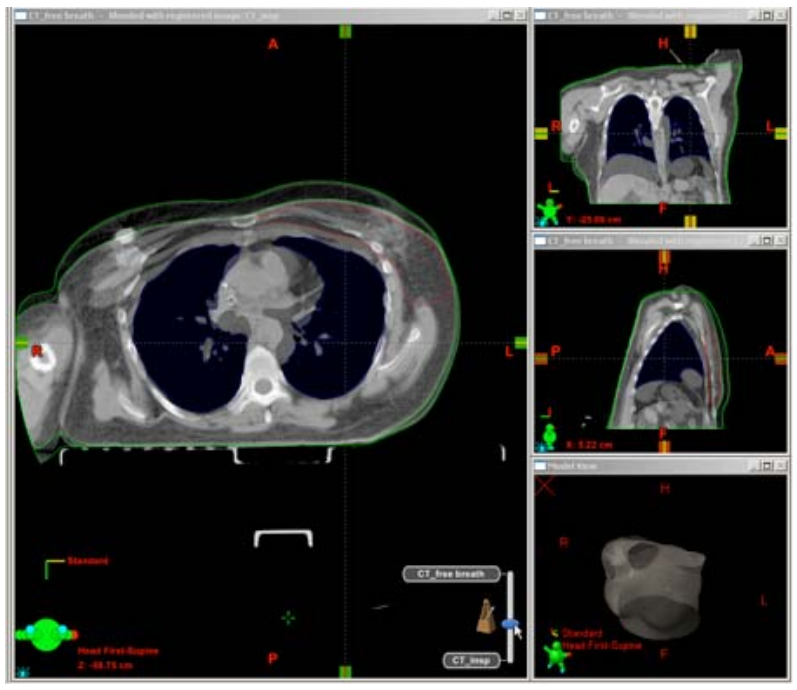

Figure 1. FB and DI CT images registration according to DICOM coordinates. Lung volume changes and targets replacement between FB and DI.

touring atlas [16]. The target volumes were the breast or chest wall, lymph nodes namely level I, II, III axillaries, supraclaviculary and internal mammary (MI). Lung (whole, contralateral and ipsilateral), heart, left anterior descending artery (LAD) and contralateral breast were OAR. The lung volumes were automatically contoured by ECLIPSE using Hounsfield unit data and were manually corrected.

Target prescription dose was 4600 - 5000 cGy for this dosimetric study. Further planning requirements: 95\% of the target volume receiving $95 \%$ of the prescription dose (4350 - 4750 cGy) and 110\% of prescription dose (5060 5500 cGy) should not exceed 5\% of CTV volume. The dose constraints for OAR were: volume receiving $20 \mathrm{~Gy}$ (V20) $<30 \%$ for the ipsilateral lung, mean dose $3.5 \mathrm{~Gy}$ and V20 < 1\% for heart and mean dose should not exceed 1 Gy, for contralateral breast V3.5 < 1\%. Primarily, three-dimensional conformal planning was used for all patients using CT slices were taken during FB series for each patient. However planning requirements were not achieved for 4 patients. Consequently IMRT planning was done for these particular 4 patients. Then, all plans were exported to DI and EE image series to obtain that dose-volume information changes depending on respiratory motion. Any parameter difference such as beam angles, wedges, field size, etc. of FB plan was not allowed in planning for DI and EE series. In order to avoid the effect of heterogeneity difference during recalculation, $\mathrm{MU}$ values from FB plan were entered for each field, in DI and EE plan. Modified Batho heterogeneity correction algorithm was on during all calculations.

The absolute and percentages of whole, ipsilateral and contralateral lung V5, V20 and prescription dose (PD) volumes belong to different breath cycle namely FB, DI 
and EE were calculated and compared each other. Whether there was a difference between V20 among breath cycles was studied. Furthermore, target coverage, min, mean and max targets doses for different breath cycles namely FB, DI and EE were calculated and compared each other. Parts of this study examining hearth and contralateral breast volume-dose changes during breath cycle were published and being published elsewhere separately. The significance of dose and volume changes was investigated using Wilcoxon test (PASW statistics 18) [13].

\section{Results}

The absolute and percentages of V5, V20 and PD volumes of whole, ipsilateral and contralateral lung during FB, DI and EE are listed in Table 1. The differences between FB-DI were found significant for criteria namely absolute whole, ipsilateral and contralateral lung volumes, whole lung V5, ipsilateral lung V5, whole lung V20, lung volume receiving $\mathrm{PD}$, ipsilateral lung volume receiving PD, $\%$ lung volume receiving PD and \% ipsilateral lung volume receiving $\mathrm{PD}$. However, contralateral lung volume was the only significant factor between FB-EE (Figure 2).

Calculated min, max and mean target namely whole breast, axillary level I-III, supraclaviculary, infraclaviculary and MI for different breath cycle and are shown in the Table 2. Average targets coverage was adequate for all breath cycle while target coverage was not adequate for 6 out of 10 patients. No significant difference was found between whole breast doses. However there was a significant differences between mean MI and level III axillary lymph node doses belongs to FB and DI and mean level II axillary doses belongs to FB and EE (Figure 3).

\section{Discussion}

In the present study, there were significant differences between FB-DI for absolute whole, ipsilateral, contralateral, V5, V20 lung volumes and volume exposed PD as reported previously $[13,14]$. On the other hand, absolute lung volumes were not significantly differ between FB and EE except contralateral lung volume. As known, absolute lung volume increases during inspiration in comparison to FB, because lung inflates. Percent lung volume is better than absolute volume to estimate radiation related side effects. Both exposed dose and \% volume are determine radiation side effects $[17,18]$. Lung functions permanently damage when exposed radiation doses greater than 20 Gy which is accepted as tolerance dose for lung [16]. Recently, Stranzl et al. reported significant dose decrease in both exposed heart and lung in their study examining benefit of radiotherapy during deep inspirium breath-hold in patients with breast carci-

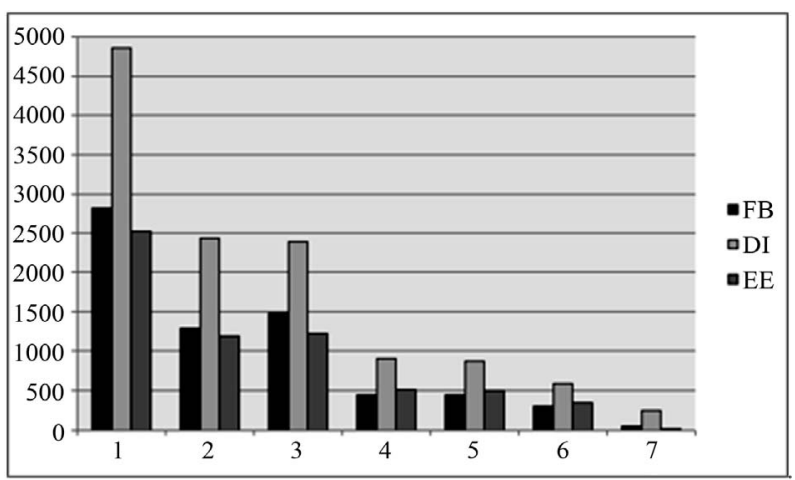

(a)

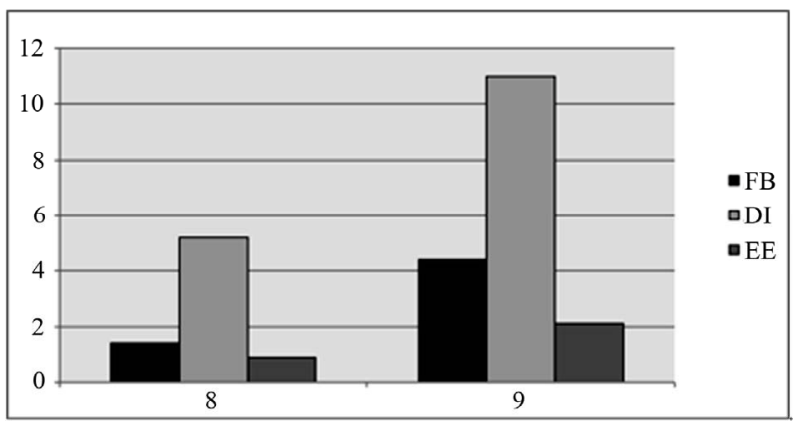

(b)

Data are shown as median values; 1) Whole lung volume (ml); 2) Ipsilateral lung volume (ml); 3) Contralateral lung volume (ml); 4) Lung volume receiving 5 Gy (ml); 5) Ipsilateral lung volume receiving 5 Gy (ml); 6) Lung volume receiving $20 \mathrm{~Gy}(\mathrm{ml}) ; 7$ ) Lung volume receiving prescription dose (ml); 8) \% Lung volume receiving prescription dose; 9) \% Ipsilateral lung volume receiving prescription dose.

Figure 2. (a) Absolute lung volume and exposed absolute lung volume; (b) Percentage of exposed lung volume differences during breath cycle.

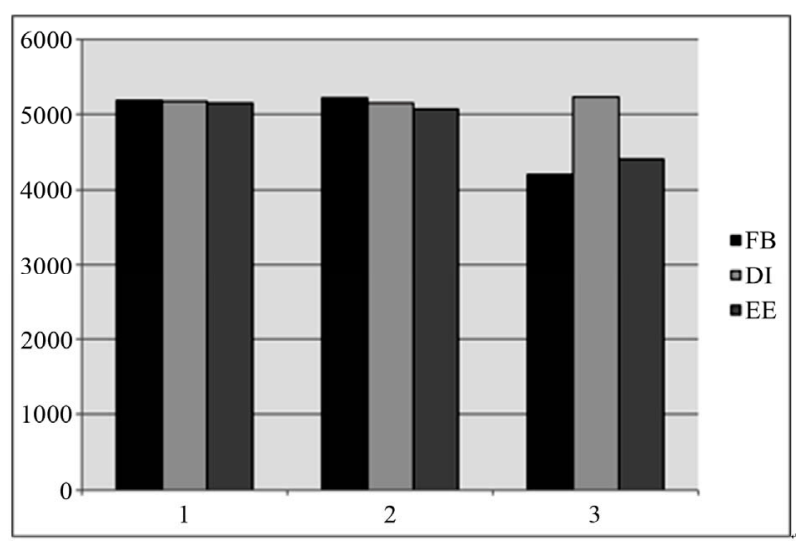

Data are shown as median values; 1) Mean axillary (Level III) dose (cGy); 2) Mean (Supraclaviculary) dose (cGy); 3) Mean (MI) dose.

Figure 3. Target (lymph nodes) dose differences according to breath cycle.

noma receiving RT [19]. They reported significant decrease in \% ipsilateral lung V20 during deep inspirium breath-hold, average ipsilateral V20 values changes \% 2.5 for whole group. However decrease were seen in 8 
Table 1. Organ at risk dose and volume variations according to breath cycles. Significant of differences were shown as " $p$ " and " $p$ " " values representing variation between FB-DI and F B-EE respectively.

\begin{tabular}{|c|c|c|c|c|c|}
\hline & FB & DI & $p^{*}$ & $\mathbf{E E}$ & $p^{* *}$ \\
\hline & Med (max-min) & Med (max-min) & & Med (max-min) & \\
\hline Whole lung volume (ml) & $2822.5(4134-1722)$ & $4863(6019$ - 1665) & 0.007 & $2525.5(3950.2-1710)$ & 0.059 \\
\hline Ipsilateral lung volume (ml) & $1289(1823-853)$ & $2443.5(2753-825)$ & 0.007 & $1189(1725.1$ - 738) & 0.083 \\
\hline Contralateral lung volume (ml) & $1478.5(2311-871)$ & $2389.5(3247-840)$ & 0.074 & $1219.5(2224.5$ - 774) & 0.007 \\
\hline Lung volume receiving $5 \mathrm{~Gy}(\mathrm{ml})$ & 451.5 (1279 - 365) & $904.5(1789-355)$ & 0.007 & $508.5(1166.8-367)$ & 0.646 \\
\hline$\%$ Lung volume receiving 5 Gy & $20.2(31-14)$ & $20(31.5-15)$ & 0.440 & $21.7(29.6-14.6)$ & 0.066 \\
\hline Ipsilateral lung volume receiving $5 \mathrm{~Gy}(\mathrm{ml})$ & $451.5(1256-366)$ & $873.5(1676-0)$ & 0.037 & $501(1158$ - 367) & 0.646 \\
\hline Lung volume receiving $20 \mathrm{~Gy}(\mathrm{ml})$ & $304.5(598-230)$ & $593(1053-261)$ & 0.007 & $345(582-234)$ & 0.799 \\
\hline \% Lung volume receiving 20 Gy & $13.1(17.6-8.3)$ & $13.9(17.5-10)$ & 0.109 & $14.1(19.6-9)$ & 0.308 \\
\hline$\%$ Ipsilateral lung volume receiving $20 \mathrm{~Gy}$ & $30.4(41.5-19)$ & $29.2(38-22)$ & 0.721 & $29.4(40.2-20)$ & 0.200 \\
\hline Lung volume receiving prescription dose (ml) & $50.3(147-4)$ & $244.8(371$ - 100) & 0.007 & $23(174-0)$ & 0.959 \\
\hline$\%$ Lung volume receiving prescription dose & $1.4(5.8-0.1)$ & $5.2(7-2.5)$ & 0.005 & $0.9(6.2-0)$ & 0.444 \\
\hline \% Ipsilateral lung volume receiving prescription dose & $4.4(11.8-0.1)$ & $11(15-5.4)$ & 0.005 & $2.1(12,5-0)$ & 0.508 \\
\hline
\end{tabular}

" $p$ ", and " $p$ **, values represent the significance; (FB) free breathing, (DI) deep inspiration, (EE) end of expiration.

Table 2. Target dose differences according to breath cycle. Significant of differences were shown as " $p$ " and " $p$ " values representing variation between FB-DI and FB-EE respectively.

\begin{tabular}{cccccc}
\hline & FB & DI & $\boldsymbol{p}^{*}$ & EE & $\boldsymbol{p}^{\text {*** }}$ \\
\hline & Med (max-min) & Med (max-min) & & Med (max-min) \\
\hline Mean breast dose (cGy) & $5097.5(5176-4913)$ & $5128.5(5573.4-4677)$ & 0.203 & $5026(5250-507)$ & 0.114 \\
Mean (Level I) dose (cGy) & $4907.5(5146-4627)$ & $4923(5082-4693)$ & 0.333 & $4898.5(5045-4616)$ & 0.169 \\
Mean (Level II) dose (cGy) & $5032(5300-4740)$ & $5112(5407-4426)$ & 0.575 & $5016.5(5306-4574)$ & 0.760 \\
Mean (Level III) dose (cGy) & $5181.5(5341-4886)$ & $5179.5(5423-5091)$ & 0.047 & $5154.5(5365-4983)$ & 0.203 \\
Mean (Supraclaviculary) dose (cGy) & $5217(5409-4981)$ & $5147(5430-4900)$ & 0.203 & $5070.5(5331-4634)$ & 0.009 \\
Mean (MI) dose (cGy) & $4205.5(5409-2418)$ & $5232(6164-5026)$ & 0.011 & $4412.5(5210-2446)$ & 0.721 \\
\hline
\end{tabular}

" $p$ "” and " $p$ "*, values represent the significance; (FB) free breathing, (DI) deep inspiration, (EE) end of expiration.

out of 11 patients. Likewise, Vikstrom et al. reported significant ipsilateral lung V20 decrease during breathhold in their study, which is searching for heart, and lung dose changes during breath-hold in patients with breast carcinoma receiving RT. Yet, the amount of decrease was limited to $2.2 \%$ [13]. In present study breath cycle did not have a significant effect on \% ipsilateral lung V20 while there was $1.2 \%$ average decrease in DI in comparison to FB. Decreases were seen 5 out of 10 patients. One speculates that, there was a tendency to decrease in ipsilateral lung V20, but this difference was not statistically significant. Target selection differences could be the reason of dissimilar result. Whole breastchest wall and all regional lymph nodes were within target in present study while whole breast and MI alongside whole breast was targeted in Stranzl study and whole breast was only targeting in Vikstrom study.
IMRT increases low dose area arising scattering radiation doses, have been used more common in breast carcinoma because of its benefit of heart and lung protection [20]. Then V5 became an issue, yet the long term effect on lung function is not known. As seen on table, there were no significant differences between V5 and V20. There was a tendency to increase \% lung volume exposed to prescription dose. However the amount of difference is limited.

According to the results of this study, the ipsilateral lung V20 is approximately $28 \%-29 \%$ in all phases of breathing. This result is compatible with previous studies [1]. Radiation Therapy Oncology Group recommends that, ipsilateral V20 volumes should be restricted to $10 \%$ patients receiving radiotherapy for "breast only" and $30 \%$ for "breast plus regional lymph nodes" respectively [21-23]. 
Individual organ motion could affect treatment planning, target dose and determined dose limits. Therefore, organ motion should be considered and treatment planning tailored accordingly. Dose differences between plans using FB and DI images arise both from organ motion and heterogeneity differences. It would be ideal for the organ position to be constant during planning and the course of treatment. Respiratory-gated radiotherapy could help to obtain an even level during the breath cycle and achieve treatment execution within the determined dose limits.

Because patients with breast carcinoma have higher life expectancy, side effects are a very important factor to be considered. Lung side effects could manifest months to years after completion of radiotherapy [3]. Although there are some approaches to relieve symptoms, chronic breathing problems related to radiation therapy are ireversible and progressive. They could affect the patient's quality of life. Lung side effects can be particularly serious in patients receiving a combination of chemotherapy and radiotherapy. Therefore these patients should be followed-up carefully.

Although there were dose differences for some targets, except for MI lymph nodes, these changes were limited to $200 \mathrm{cGy}$, during different phases of the respiration cycle. However the MI lymph nodes are required to irradiate only in selected higher stage cases. For example, in this study, those differences were studied; however, MI lymph nodes were treated with only 1 out of 10 patients.

\section{Conclusion}

The results of this study suggest that, heterogeneity correction without considering breath control is adequate to estimate the target dose during the whole breath cycle. Furthermore, radiotherapy planning to use FB is sufficient to estimate lung side effects. Because there is no significant difference in V20 values for planning images obtained in the FB, DI and EE phases.

\section{REFERENCES}

[1] M. S. Moran and B. G. Haffty, "Radiation Techniques and Toxicities for Locally Advanced Breast Cancer," Seminars in Radiation Oncology, Vol. 19, No. 4, 2009, pp. 244-255. doi:10.1016/j.semradonc.2009.05.007

[2] T. Rancati, B. Wennberg, P. Lind and G. Galiardi, "Early Clinical and Radiological Pulmonary Complications Following Breast Cancer Radiation Therapy: NTCP Fit with Four Different Models," Radiotherapy \& Oncology, Vol. 82, No. 3, 2007, pp. 308-316. doi:10.1016/j.radonc.2006.12.001

[3] L. B. Marks, S. M. Bentzen, J. O. Deasy, F. M. Kong, J. D. Bradley, I. S. Vogelius, et al., "Radiation Dose-Volume Effects in the Lung," International Journal of Radiation Oncology, Biology, Physics, Vol. 76, No. 3, 2010, pp. 70-76. doi:10.1016/j.ijrobp.2009.06.091

[4] L. B. Marks, D. Hollis, M. Munley, G. Bentel, M. Garipagaoglu, M. Fan, et al., "The Role of Lung Perfusion Imaging in Predicting the Direction of Radiation-Induced Changes in Pulmonary Function Tests,” Cancer. Vol. 88, No. 9, 2000, pp. 2135-2141.

doi:10.1002/(SICI)1097-0142(20000501)88:9<2135::AID -CNCR20>3.0.CO;2-H

[5] E. D. Yorke, A. Jackson, K. E. Rosenzweig, L. Braban, S. A. Leibel and C. C. Ling, "Correlation of Dosimetric Factors and Radiation Pneumonitis for Non-Small-Cell Lung Cancer Patients in a Recently Completed Dose Escalation Study," International Journal of Radiation Oncology, Biology, Physics, Vol. 63, No. 3, 2005, pp. 672-682. doi:10.1016/j.ijrobp.2005.03.026

[6] J. D. Bradley, A. Hope, I. El Naqa, A. Apte, P. E. Lindsay, W. Bosch, et al., "A Nomogram to Predict Radiation Pneumonitis, Derived from a Combined Analysis of RTOG 9311 and Institutional Data,” International Journal of Radiation Oncology, Biology, Physics, Vol. 69, No. 4, 2007, pp. 985-992. doi:10.1016/j.ijrobp.2007.04.077

[7] M. L. Hernando, L. B. Marks, G. C. Bentel, M. N. Zhou, D. Hollis, S. K. Das, et al., "Radiation-Induced Pulmonary Toxicity: A Dose-Volume Histogram Analysis in 201 Patients with Lung Cancer,” International Journal of Radiation Oncology, Biology, Physics, Vol. 51, No. 3, 2001, pp. 650-659. doi:10.1016/S0360-3016(01)01685-6

[8] Y. Seppenwoolde, K. D. Jaeger, L. J. Boersma, S. A. Belderbos and J. V. Lebesque, "Regional Differences in Lung Radiosensitivity after Radiotherapy for NonsmallCell Lung Cancer,” International Journal of Radiation Oncology, Biology, Physics, Vol. 60, No. 3, 2004, pp. 748-758. doi:10.1016/j.ijrobp.2004.04.037

[9] V. M. Remouchamps, F. A. Vicini, M. B. Sharpe, L. L. Kestin, A. A. Martinez and J. W. Wong, "Significant Reductions in Heart and Lung Doses Using Deep Inspiration Breath Hold with Active Breathing Control and Intensity-Modulated Radiation Therapy for Patients Treated with Loco Regional Breast Irradiation,” International Journal of Radiation Oncology, Biology, Physics, Vol. 55, No. 2, 2003, pp. 392-406. doi:10.1016/S0360-3016(02)04143-3

[10] A. N. Pedersen, S. Korreman, H. Nyström and L. Specth, "Breathing Adapted Radiotherapy of Breast Cancer: Reduction of Cardiac and Pulmonary Doses Using Voluntary Inspiration Breath-Hold,” Radiotherapy \& Oncology, Vol. 72, No. 1, 2004, pp. 53-60. doi:10.1016/j.radonc.2004.03.012

[11] P. M. K. Leung, B. Seaman and P. Robinson, "Low-Density Inhomogeneity Corrections for 22-MeV X-Ray Therapy,” Radiology, Vol. 94, No. 2, 1970, p. 449.

[12] R. O. Kornelson and M. E. J. Young, "Changes in the Dose-Profile of a 10 MV X-Ray Beam within and beyond Low Density Material,” Medical Physics, Vol. 9, No. 1, 1982, p. 114. doi:10.1118/1.595059

[13] J. Vikström, M. H. B. Hjelstuen, I. Mjaaland and K. I. Dybvik, "Cardiac and Pulmonary Dose Reduction for Tangentially Irradiated Breast Cancer, Utilizing Deep Inspiration Beath-Hold with Audio-Visual Guidance, with- 
out Compromising Target Coverage,” Acta Oncologica, Vol. 50, No. 1, 2011, pp. 42-50.

[14] R. C. Frazier, F. A. Vicini, M. B. Sharpe, D. Yan, J. Fayad, K. L. Baglan, et al., "Impact of Breathing Motion on Breast Radiotherapy: A Dosimetric Analysis Using Active Breathing Control," International Journal of Radiation Oncology, Biology, Physics, Vol. 58, No. 4, 2004, pp. 1041-1047. doi:10.1016/j.ijrobp.2003.07.005

[15] E. Tezcanli, E. Goksel, E. Yildiz, M. Garipagaoglu, O. Senkesen, H. Kucucuk, et al., "Does Radiotherapy Planning without Breath Control Compensate Intra-Fraction Heart and Its Compartments' Movement?” Breast Cancer Research and Treatment, Vol. 126, No. 1, 2011, pp. 8592. doi:10.1007/s10549-010-1306-0

[16] X. A. Li, A. Tai, D. W. Arthur, T. A. Buchholz, S. MacDonald, L. B. Marks, et al., "Variability of Target and Normal Structure Delineation for Breast-Cancer Radiotherapy: A RTOG Multi-Institutional and Multi-Observer Study," International Journal of Radiation Oncology, Biology, Physics, Vol. 73, No. 3, 2009, pp. 944-951. doi:10.1016/j.ijrobp.2008.10.034

[17] T. I. Lingos, A. Recht, F. Vicini, A. Abner, B. Silver and J. R. Harris, "Radiation Pneumonitis in Breast Cancer Patients Treated with Conservative Surgery and Radiation Therapy," International Journal of Radiation Oncology, Biology, Physics, Vol. 21, No. 2, 1991, pp. 355-360. doi:10.1016/0360-3016(91)90782-Y

[18] A. G. Taghian, S. I. Assaad, A. Niemierko, I. Kuter, J. Younger, R. Schoenthaler, et al., "Risk of Pneumonitis in Breast Cancer Patients Treated with Radiation Therapy and Combination Chemotherapy with Paclitaxel,” Jour- nal of the National Cancer Institute, Vol. 93, No. 23, 2001, pp. 1806-1811. doi:10.1093/jnci/93.23.1806

[19] H. Stranzl, B. Zurl, T. Langsenlehner, K. S. Kapp, "Widetangential Fields Including the Internal Mammary Lymph Nodes in Patients with Left-Sided Breast Cancer," Strahlentherapie und Onkologie, Vol. 185, No. 2, 2009, pp. 155-160. doi:10.1007/s00066-009-1939-2

[20] E. J. Hall, C. S. Wuu, "Radiation-Induced Second Cancers: The Impact of 3D-CRT and IMRT," International Journal of Radiation Oncology, Biology, Physics, Vol. 56, No. 1, 2003, pp. 83-88. doi:10.1016/S0360-3016(03)00073-7

[21] G. Gagliardi, J. Bjohle, I. Lax, A. Ottolenghi, F. Eriksson, A. Liedberg, et al., "Radiation Pneumonitis after Breast Cancer Irradiation: Analysis of the Complication Probability Using the Relative Seriality Model,” International Journal of Radiation Oncology, Biology, Physics, Vol. 46, No. 2, 2000, pp. 373-381. doi:10.1016/S0360-3016(99)00420-4

[22] N. Wolmark, W. J. Curran, F. Vicini, J. White, J. P. Costantino, D. Arthur, et al., "Toxicity of Three-Dimensional Conformal Radiotherapy for Accelerated Partial Breast Irradiation,” International Journal of Radiation Oncology, Biology, Physics, Vol. 75, No. 3, 2009, pp. 1290 1296.

[23] A. J. Hope, P. E. Lindsay, I. El Naqa, J. R. Alaly, M. Vicic, J. D. Breadley, et al., "Modeling Radiation Pneumonitis Risk with Clinical, Dosimetric, and Spatial Parameters," International Journal of Radiation Oncology, Biology, Physics, Vol. 65, No. 1, 2006, pp. 112-124. doi:10.1016/j.ijrobp.2005.11.046 